\title{
Safety, Efficacy, and Prognostic Factors After Radioembolization of Hepatic Metastases from Breast Cancer: A Large Single-Center Experience in 81 Patients
}

\author{
Wolfgang P. Fendler ${ }^{1}$, Hanna Lechner ${ }^{1}$, Andrei Todica ${ }^{1}$, Karolin J. Paprottka ${ }^{2}$, Philipp M. Paprottka ${ }^{2}$, Tobias F. Jakobs ${ }^{3}$, \\ Marlies Michl ${ }^{4}$, Peter Bartenstein ${ }^{1,5}$, Sebastian Lehner ${ }^{* 1}$, and Alexander R. Haug*1,6 \\ ${ }^{1}$ Department of Nuclear Medicine, Ludwig-Maximilians-University of Munich, Munich, Germany; ${ }^{2}$ Department of Clinical \\ Radiology, Ludwig-Maximilians-University of Munich, Munich, Germany; ${ }^{3}$ Department of Diagnostic and Interventional Radiology, \\ Hospital Barmherzige Brueder, Munich, Germany; ${ }^{4}$ Department of Hematology and Oncology, Ludwig-Maximilians-University of \\ Munich, Munich, Germany; ${ }^{5}$ Comprehensive Cancer Center Munich (CCCM), Ludwig-Maximilians-University of Munich, Munich, \\ Germany; and ${ }^{6}$ Division of Nuclear Medicine, Medical University of Vienna, Vienna, Austria
}

The present study evaluated safety, efficacy, and prognostic factors for ${ }^{90}$ Y-yttrium microsphere radioembolization of unresectable liver metastases from breast cancer. Methods: Eighty-one patients were treated with radioembolization. Acute toxicity was monitored through daily physical examination and serum tests until $3 \mathrm{~d}$ after radioembolization; late toxicity was evaluated until $12 \mathrm{wk}$ after radioembolization. Overall survival and response according to ${ }^{18} \mathrm{~F}-\mathrm{FDG}$ PET $(>30 \%$ decrease of tracer uptake) and CA15-3 serum level (any decline) were recorded. Pretherapeutic characteristics, including pretreatment history, liver function tests, and PET/CT parameters, were assessed by univariate and subsequent multivariate Cox regression for predicting patient survival. Results: A toxicity grade of 3 or more based on clinical symptoms, bilirubin, ulcer, pancreatitis, ascites, or radioembolization-induced liver disease occurred in $10 \%$ or less of patients. Two patients eventually died from radioembolization-induced liver disease. Sequential lobar treatment and absence of prior angiosuppressive therapy were both associated with a lower rate of serious adverse events. On the basis of PET/CA15-3 criteria, $52 / 61 \%$ of patients responded to treatment. Median overall survival after radioembolization was $35 \mathrm{wk}$ (interquartile range, $41 \mathrm{wk}$ ). Pretherapeutic tumor burden of the liver greater than $50 \%$ or more $(P<0.001$; hazard ratio, $5.67 ; 95 \%$ confidence interval, 2.41-13.34) and a transaminase toxicity grade of 2 or more $(P=0.009$; hazard ratio, 2.15; 95\% confidence interval, 1.21-3.80) independently predicted short survival. Conclusion: Radioembolization for breast cancer liver metastases shows encouraging local response rates with low incidence of serious adverse events, especially in those patients with sequential lobar treatment or without prior angiosuppressive therapy. High hepatic tumor burden and liver transaminase levels at baseline indicate poor outcome.

Key Words: radioembolization; hepatic metastases; breast cancer; survival

J Nucl Med 2016; 57:517-523

DOI: 10.2967/jnumed.115.165050

Received Aug. 4, 2015; revision accepted Nov. 25, 2015.

For correspondence or reprints contact: Wolfgang Peter Fendler, Klinik und

Poliklinik für Nuklearmedizin, Marchioninistrasse 15, 81377 Munich, Germany.

E-mail: wolfgang.fendler@med.uni-muenchen.de

${ }^{*}$ Contributed equally to this work.

Published online Jan. 7, 2016.

COPYRIGHT (c) 2016 by the Society of Nuclear Medicine and Molecular Imaging, Inc.
B reast cancer is the most common malignancy in women other than nonmelanoma skin tumors. Despite advances in early detection and treatment, a significant proportion of breast cancer patients develop metastases during the course of their disease (1). Median survival in patients with liver metastases from breast cancer has been estimated to be commonly poor (2). In advanced cases, curative resection of liver metastases cannot be performed because of a multisegmental involvement (3). Radioembolization using ${ }^{90}$ Y-yttrium microspheres has emerged as a palliative treatment option in patients with unresectable liver-dominant disease. Response rates between $39 \%$ and $51 \%$ have been reported after radioembolization in patients with breast cancer liver metastases (BRCLM) (4-6). However, radioembolization is associated with adverse events, which range in severity from a mild postembolization syndrome, including fatigue, abdominal pain, nausea, or fever, to serious complications such as radioembolization-induced liver disease (REILD), pancreatitis, or gastrointestinal ulceration $(7,8)$. Indeed, lethal outcomes after radioembolization for BRCLM have been reported $(9,10)$. Radioembolization is performed at a stage of terminal disease, with an aim to prolong patient survival without limiting quality of life. In deciding to administer radioembolization, the advantages of this additional treatment have to be carefully weighed against disadvantages, such as complications or further hospitalization. A better knowledge of the efficacy and safety of radioembolization will help clinicians to carefully select suitable candidates to minimize the risk of treatment-related side effects. Moreover, indicators of poor outcome will help to estimate a patient's prognosis before radioembolization is performed. The aim of the present retrospective single center study was to analyze the efficacy and safety of radioembolization in a large cohort of patients with liver metastases from breast cancer. Furthermore, clinical characteristics, radiologic findings, and laboratory tests were examined for their accuracy to predict patient survival after radioembolization.

\section{MATERIALS AND METHODS}

\section{Patients}

${ }^{90}$ Y-yttrium microsphere radioembolization was performed between March 2003 and August 2013 in the Department of Nuclear Medicine, University Hospital of the Ludwig-Maximilians-University Munich. The inclusion criteria were similar to those reported in our previous studies $(11,12)$ : age, more than $18 \mathrm{y}$; unresectable, progressive 
BRCLM refractory to systemic therapy; no evidence of a second malignancy of different origin at time of radioembolization; preserved liver function, as defined by a serum total bilirubin of $2.0 \mathrm{mg} / \mathrm{dL}$ or less; performance status of functional impairment of $60 \%$ or greater as measured with the Karnofsky index (13); preradioembolization life expectancy of at least $3 \mathrm{mo}$; and fitness to undergo angiography. Patients with limited extrahepatic metastases were not excluded if the hepatic metastases were deemed to be the predominant and presumably life-limiting tumor site. Exclusion criteria were liver failure, evidence of any uncorrectable hepatic arterial blood flow to the gastrointestinal tract, pulmonary shunt from the hepatic artery greater than $20 \%$, or complete portal venous occlusion as described previously (6). A flow diagram for selection of the study cohort is shown in Supplemental Figure 1 (supplemental materials are available at http://jnm.snmjournals.org). All patients gave written consent to undergo radioembolization. The observation period for overall patient survival ended on August 30, 2014. The retrospective study protocol was approved by the local ethics committee, and written informed consent for entry into the study was waived.

\section{Radioembolization}

Fifty-eight patients underwent whole-liver (WL) radioembolization by application of SIR-spheres (SIRTEX Medical) directly into the right and left hepatic artery in a single session (single WL group) as reported previously (6). In 23 patients, lobes were treated separately (separate lobar [SL] group): the liver lobe presenting a higher tumor burden in preradioembolization imaging was treated first; in 15 of these 23 patients, radioembolization of the remaining lobe was administered after a 4-wk interval. The second radioembolization was not performed in the case of aberrant vessels $(n=1)$ or absence of tumor $(n=7)$. In 54 of $81(67 \%)$ patients, the administered activity of microspheres was calculated using the body surface area method (14): activity in $\mathrm{GBq}=$ (body surface area -0.2$)+($ liver involvement in \%/100). In earlier cases $(n=27$ of $81,33 \%)$, dose was determined using the empiric method $(2.0 \mathrm{GBq}$ when $<25 \%$ involvement; $2.5 \mathrm{GBq}$ when $>25 \%$ involvement) (14). None of the patients received REILD prophylaxis with methyl-prednisolone or ursodeoxycholic acid.

\section{Imaging and Pretherapeutic Examinations}

Within 1-4 wk before radioembolization, all patients underwent angiography with application of $100 \mathrm{MBq}$ of $99 \mathrm{~m}$ Tc-MAA (GE Healthcare) directly into the right and left hepatic artery to identify any relevant aberrant vessel and to calculate the shunt fraction of labeled microspheres to the lung. Prophylactic coiling of extrahepatic arteries was performed, if deemed necessary. Imaging with wholebody ${ }^{18} \mathrm{~F}-\mathrm{FDG}$ PET/CT was performed using a Gemini scanner (Philips) or a Biograph 64 TruePoint PET/CT scanner (Siemens Healthcare) as described previously (6). The tumor-to-liver volume ratio was categorized on $\mathrm{CT}$ images by 2 authors with together more than $15 \mathrm{y}$ of experience in PET/CT interpretation into $<25 \%$, $25 \%-50 \%$, or $\geq 50 \%$. On admission, $1 \mathrm{~d}$ before radioembolization, serum tests for liver function (including bilirubin, alanine transaminase [ALT], aspartate transaminase [AST], $\gamma$-glutamyl transpeptidase [GGT]), coagulation profiles, metabolic panel, blood count, and the tumor markers carcinoembryonic antigen, and CA15-3 were obtained.

\section{Assessment of Toxicity}

After radioembolization, all patients were monitored for $3 \mathrm{~d}$ as inpatients for acute toxicity by daily physical examination; interview; and serum tests of liver function, coagulation profiles, and metabolic panel. Patients with worsening symptoms or liver function tests were not discharged before substantial improvement. Late toxicity was evaluated in all patients at $12 \mathrm{wk}$ after first radioembolization by physical examination, standardized interview, and serum tests including tumor marker levels. The National Cancer Institute's Common Terminology
Criteria for Adverse Events (CTCAE; version 4.0) were used (15). REILD was defined as the presence of a serum total bilirubin of $3.0 \mathrm{mg} / \mathrm{dL}$ or greater and a grade of 2 or more ascites within 3 mo after radioembolization, in the absence of tumor progression or bile duct obstruction. A toxicity grade of 3 or more, including occurrence of ulcer, pancreatitis, and REILD, was defined as a serious adverse event (SAE). The schedule for radioembolization and follow-up visits is illustrated in Supplemental Figure 2.

\section{Assessment of Response and Survival}

The primary endpoint was overall survival (OS) after radioembolization. OS was defined as the interval between date of radioembolization until the last date of contact as censored observation or until diseaserelated death as the event of interest. Secondary endpoints were response by ${ }^{18} \mathrm{~F}-\mathrm{FDG}$ PET or serum tumor marker level. ${ }^{18} \mathrm{~F}-\mathrm{FDG}$ PET/CT was performed in 56 of 81 (69\%) patients at the time of late follow-up. PET response was defined as a greater than $30 \%$ decrease of $\mathrm{SUV}_{\max }$ from baseline to follow-up determined in up to 5 lesions in accordance with our previous report (6). In patients with CA15-3 serum level above the upper limit of normal at baseline, tumor marker response was defined by any decrease in CA15-3 level at the time of late follow-up based on findings from Guadagni et al. (16). One subgroup $(n=30)$ underwent continuous surveillance by ${ }^{18} \mathrm{~F}$-FDG PET/CT at 3-mo intervals after radioembolization until death. We defined time-to-liver-progression $\left(\mathrm{TTP}_{\text {liver }}\right)$ in this group as the time interval between date of radioembolization until any increase of at least $30 \%$ in $\mathrm{SUV}_{\max }$ in at least 1 liver lesion relative to baseline.

\section{Statistical Analysis}

Quantitative data are presented as total number (percentage) or median (interquartile range [IQR]). Kaplan-Meier analysis and the $\log$ rank test were used for comparison of survival rates. Prior systemic therapy, in 8 subgroups defined by differing antineoplastic mechanism of action, was tested for association with toxicity by odds ratio (OR) and the $\chi^{2}$ test. After Pearson intercorrelation was excluded, univariate Cox regression analysis was performed to examine the association between pretherapeutic parameters and OS. Any parameter with a $P$ value of less than 0.20 in the univariate analysis was entered into a multivariate Cox regression model. The Wald stepwise selection method with a $P$ value of 0.05 as entry probability and a $P$ value of 0.10 as removal probability was applied in the multivariate analysis. Hazard ratio with 95\% confidence interval was calculated to quantify the strength of the association between parameters and OS. A statistically significant difference was defined as a $P$ value of less than 0.05 . The SPSS software package (version 15.0; SPSS Inc.) was used for all statistical analysis.

\section{RESULTS}

\section{Patients}

Patient characteristics are given in Table 1. At the time of radioembolization, patients presented with a high rate of extrahepatic metastases $(54 / 81,67 \%)$. Presence of extrahepatic metastases was not significantly associated with OS according to the log-rank test $(P=0.558$, Supplemental Fig. 3) and univariate regression analysis $(P=0.299)$. The median OS was $41 \mathrm{wk}(\mathrm{IQR}, 51 \mathrm{wk})$ for patients without extrahepatic metastases $(n=27), 32 \mathrm{wk}$ (IQR, $34 \mathrm{wk}$ ) for patients with bone metastases $(n=34)$, and $34 \mathrm{wk}$ (IQR, $29 \mathrm{wk}$ ) for patients with other sites (lymph node $n=3$, pancreas/ lung each $n=1)$ or multiple sites $(n=15)$ of metastases.

\section{Toxicity}

Adverse events after radioembolization are given in Table 2. On the basis of liver transaminase, SAEs were seen for 31 of $81(38 \%)$ patients; on the basis of GGT, SAEs were seen for 29 of 81 (36\%) 
TABLE 1

Baseline Characteristics of Study Cohort

\begin{tabular}{|c|c|}
\hline Characteristic $(n=81)$ & $\begin{array}{l}\text { Total or } \\
\text { median }\end{array}$ \\
\hline Age (y) & $61(15)$ \\
\hline $\begin{array}{l}\text { Time between diagnosis } \\
\text { and radioembolization (y) }\end{array}$ & $6.0(7.3)$ \\
\hline Prior local treatment & $20(25 \%)$ \\
\hline Surgery & $8(10 \%)$ \\
\hline RFA & $9(11 \%)$ \\
\hline TACE & $4(5 \%)$ \\
\hline LITT & $1(1 \%)$ \\
\hline Multiple & $2(2 \%)$ \\
\hline \multicolumn{2}{|l|}{ UICC stage at initial diagnosis } \\
\hline 1 & $4(5 \%)$ \\
\hline $2 a$ & $29(36 \%)$ \\
\hline $2 b$ & $13(16 \%)$ \\
\hline $3 a$ & $11(14 \%)$ \\
\hline $3 b$ & $8(10 \%)$ \\
\hline 4 & $16(20 \%)$ \\
\hline \multicolumn{2}{|l|}{ Tumor expression profile } \\
\hline $\mathrm{PR}+$ & $40(49 \%)$ \\
\hline $\mathrm{ER}+$ & $60(74 \%)$ \\
\hline Her2/neu+ & $28(35 \%)$ \\
\hline CA15-3 (U/mL) & $137(395)$ \\
\hline Carcinoembryonic antigen (ng/mL) & $12(28)$ \\
\hline \multicolumn{2}{|l|}{ Tumor-to-liver ratio } \\
\hline$<25 \%$ & $49(60 \%)$ \\
\hline $25 \%-50 \%$ & $24(30 \%)$ \\
\hline$\geq 50 \%$ & $8(10 \%)$ \\
\hline Radioactivity delivered (GBq) & $1.6(0.6)$ \\
\hline
\end{tabular}

RFA = radiofrequency ablation; TACE $=$ transcatheter arterial chemoembolization; LITT = laser-induced thermotherapy; UICC = International Union Against Cancer; $\mathrm{PR}+=$ progesterone receptor status positive; ER+ = estrogen receptor status positive; Her$2 /$ neu $+=$ Her-2/neu status positive.

Given are total numbers (with percentages in parentheses) or median (with IQRs in parentheses).

patients. By all other CTCAE criteria, the rate of SAE was below $10 \%$. Two patients developed pancreatitis after radioembolization, both of whom had full remission after conservative treatment. Gastrointestinal ulceration occurred in 5 patients, 4 cases of which occurred before our introduction of monitoring of hepatic arterial flow by intermittent application of contrast agent during the therapy. Bilirubin toxicity of grade 3 or 4 was observed in 8 of 81 (10\%) patients. REILD was diagnosed in 3 of $58(5 \%)$ patients after WL radioembolization in 1 session (WL group). Two of these 3 patients had further deterioration of liver function and died 11 and $12 \mathrm{wk}$ after radioembolization. The remaining patient recovered from REILD and died 70 wk after radioembolization because of tumor progression. Eight patients in the WL group had an appearance of grade 2 ascites after radioembolization, albeit without substantial bilirubin elevation (Fig. 1). None of the patients with lobar radioembolization in 2 separate sessions (SL group) experienced REILD until 3 mo after radioembolization. The rate of SAEs was lower in the SL group than WL group based on reported nausea, abdominal pain, ulcer, bilirubin, and REILD by a sum of $31 \%$ (Fig. 2). The rate of grade 1-2 nausea was $23 \%$ lower in the SL group, whereas mild abdominal pain was experienced in equal measure for both groups (74\% for SL, $67 \%$ for WL). The prior administration of monoclonal antibodies (i.e., bevacizumab alone, $n=18$; trastuzumab alone, $n=13$; both, $n=$ 4) was significantly associated with occurrence of SAE (Table 3 ), whereas the other systemic treatments were not. Two of 3 (67\%) patients with REILD, 4 of $6(67 \%)$ with grade 3 or more nausea, 5 of $7(71 \%)$ with pancreatitis or ulceration, and both patients $(100 \%)$ with grade 3 or higher abdominal pain had undergone monoclonal antibody treatment before radioembolization.

\section{Response and Survival}

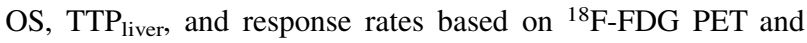
CA15-3 are presented in Table 4. The median OS of the entire cohort was $35 \mathrm{wk}$ (IQR, $41 \mathrm{wk}$ ) after radioembolization and $7.2 \mathrm{y}$ (IQR, 7.3 y) after initial diagnosis. Six patients were still alive at the end of the observation period. OS after radioembolization and diagnosis did not differ significantly between the WL and SL groups according to the log-rank test (36 [IQR, 37] vs. 29 [IQR, 45] wk; $P=0.932)$. Twenty-nine of $56(52 \%)$ patients responded to radioembolization based on ${ }^{18}$ F-FDG PET criteria. Forty-six of $81(57 \%)$ patients had an elevated CA15-3 level at baseline. Twenty-eight of these $46(61 \%)$ patients responded to radioembolization based on CA15-3 level. The median $\mathrm{TTP}_{\text {liver }}$ was $26 \mathrm{wk}$ (IQR, $18 \mathrm{wk}$ ) in the ${ }^{18} \mathrm{~F}-\mathrm{FDG}$ PET/CT surveillance group $(n=30)$.

\section{Regression Analysis}

Noncategoric variables were dichotomized on the basis of the median. Results of univariate Cox regression analysis are given in Table 5. Several variables were associated with OS in the univariate analysis and were consequently included in the multivariate analysis. Table 6 gives results of the multivariate analysis. Corresponding Kaplan-Meier survival curves are shown in Figure 3. Two variables were independently associated with significantly reduced OS after radioembolization. The relevant variables were a baseline transaminase toxicity grade of 2 or more $(P=0.009)$ and tumor-to-liver ratio of $50 \%$ or more $(P<0.001)$, which increased the risk of short survival by about 2- and 6-fold, respectively.

\section{DISCUSSION}

The aim of this study was to analyze safety, efficacy, and prognostic factors after radioembolization for hepatic metastases from breast cancer. For this purpose, we examined data from the largest series of breast cancer patients who received radioembolization for liver metastases so far. Though data were analyzed retrospectively, detailed information about the patients' medical history, adverse events, and outcome was available based on our institutional standards for patient inclusion, treatment, and follow-up. The median survival of our patients ( $35 \mathrm{wk}$ ) falls within the range of previous reports $(5,6,10)$ and did not significantly differ between patients with radioembolization of the WL in a single session versus lobar radioembolization in 2 separate sessions (36 vs. 29 wk). The 
TABLE 2

Adverse Events After Radioembolization

\begin{tabular}{|c|c|c|c|c|c|}
\hline Adverse event & All grades & Grade 1 & Grade 2 & Grade 3 & Grade 4 \\
\hline Fever & $19(23 \%)$ & 18 (22\%) & $1(1 \%)$ & - & - \\
\hline Nausea & $41(51 \%)$ & 15 (19\%) & $20(25 \%)$ & $6(7 \%)$ & - \\
\hline Abdominal pain & $58(72 \%)$ & 19 (23\%) & $37(46 \%)$ & $2(2 \%)$ & - \\
\hline Ulcer & - & - & - & $5(6 \%)$ & \\
\hline Pancreatitis & - & - & - & $2(2 \%)$ & \\
\hline Bilirubin & $50(62 \%)$ & 20 (25\%) & $22(27 \%)$ & $5(6 \%)$ & $3(4 \%)$ \\
\hline Transaminase & 80 (99\%) & $29(36 \%)$ & 20 (25\%) & $24(30 \%)$ & 7 (9\%) \\
\hline GGT & 70 (86\%) & 25 (31\%) & $16(20 \%)$ & $24(30 \%)$ & $5(6 \%)$ \\
\hline INR & 74 (91\%) & $62(77 \%)$ & $10(12 \%)$ & $2(2 \%)$ & - \\
\hline Ascites & 29 (36\%) & 20 (25\%) & $9(11 \%)$ & - & - \\
\hline REILD & - & - & - & $3(4 \%)$ & \\
\hline
\end{tabular}

liver is among the 5 leading sites of metastatic involvement, although the incidence of tumor spread is higher still in other organs such as bone or lymph nodes (17). Indeed, our patients presented with a high rate of extrahepatic metastases $(67 \%)$ but were only admitted to radioembolization when their extrahepatic tumor load was lower and presumably not life-limiting, as compared with the hepatic metastases. Even though radioembolization targets only hepatic tumor lesions, we detected no association between presence or location of extrahepatic disease and OS in our patient cohort. This supports our initial decision to include patients with limited extrahepatic metastases. About half of our patients had metabolic response by ${ }^{18} \mathrm{~F}$-FDG PET. Our rate of metabolic response falls below

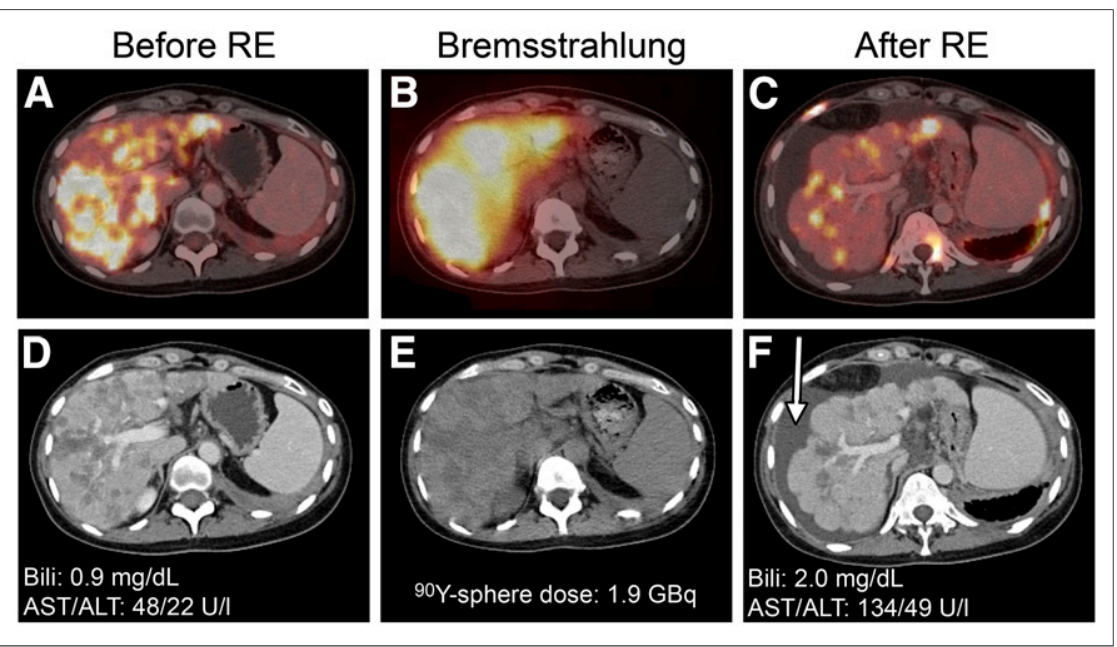

FIGURE 1. A 39-y-old female patient with new onset of ascites after radioembolization (RE) of WL in single session. Axial CT and fused ${ }^{18}$ F-FDG PET/CT images are shown at baseline (A and D) and 12 wk after radioembolization (C and F). Respective serum levels of bilirubin (Bili), AST, and ALT are given. Follow-up CT reveals signs of hepatic cirrhosis with appearance of ascites (arrow). Patient developed grade 2 toxicity for bilirubin/AST and grade 1 toxicity for ALT. Patient eventually recovered from liver toxicity but died from tumor progression at $23 \mathrm{wk}$ after radioembolization. (B and E) Axial low-dose CT (E) and fused Bremsstrahlung SPECT/CT (B) after radioembolization of WL. the rate of $84 \%$ metabolic responders reported by Gordon et al. in a subgroup of 25 patients (10). This difference may in part be explained by the definition of metabolic response, which was determined by a visual decease in glycolytic activity in 1 lesion by Gordon et al. versus a 30\% decrease in quantitative SUV in up to 5 lesions in our study. On the basis of our previous study on a subgroup of the present cohort, which showed limited prognostic value of tumor size criteria, we did not include CT-based response into our analysis (6). Median $\mathrm{TTP}_{\text {liver }}$ was 26 wk in our subgroup of patients with ${ }^{18} \mathrm{~F}-\mathrm{FDG}$ PET/CT surveillance after radioembolization; this result for hepatic progression is consistent with the 13- to 27-wk range of time-to-progression reported in 2 previous studies $(10,18)$. Such prolonged progression-free intervals emphasize the potential of radioembolization to halt or slow hepatic progression in patients with chemorefractory BRCLM.

Previous trials, including 1 study from our department, reported a high incidence of postembolization syndrome $(67 \%$ and $100 \%)$ after radioembolization for $\operatorname{BRCLM}(5,9)$. In our expanded cohort, we likewise found mild to moderate symptoms of fever, nausea, or abdominal pain in $23 \%-72 \%$ of patients. However, radioembolization was also associated with severe adverse events: 3 of 81 (4\%) patients developed REILD, a lifethreatening condition. Indeed, 2 of these 3 patients died within $12 \mathrm{wk}$ from liver toxicity as compared with an overall median survival of $35 \mathrm{wk}$ after radioembolization. The present incidence of high-grade toxicity was also comparable to rates given in the literature: SAEs occurred in $10 \%$ of patients based on bilirubin. Similarly, Gordon et al. and Bangash et al. found grade 3 or higher bilirubin toxicity in $6 \%$ and $11 \%$ of their patients $(4,10)$. SAEs occurred in $39 \%$ of our patients based on liver transaminase 


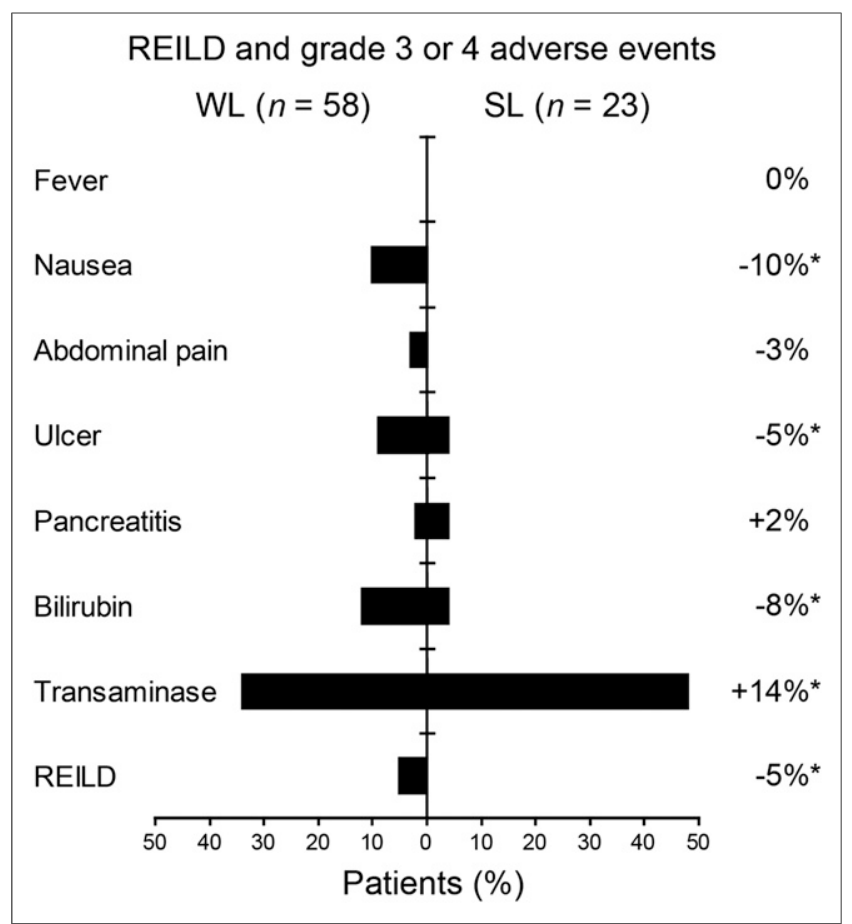

FIGURE 2. Incidence of adverse events by treatment group $(n=81)$. Difference between treatment groups (i.e., incidence toxicity SL minus $\mathrm{WL}$ ) is depicted, with those exceeding $5 \%$ marked with *.

and in $36 \%$ based on GGT. Elevation of liver enzymes, however, was only transient, and serum levels declined to a low grade of toxicity in most patients after 3 mo. Indeed, only 9 of $81(11 \%)$ patients had grade 3 to 4 elevation of liver enzymes at the time of

TABLE 3

Therapy-Associated Risk for SAE After Radioembolization

\begin{tabular}{lcccccc}
\hline & & & & \multicolumn{3}{c}{$\begin{array}{c}95 \% \text { confidence } \\
\text { interval }\end{array}$} \\
\cline { 5 - 7 } Variable & $n$ & $P$ & $\begin{array}{c}\text { Odds } \\
\text { ratio }\end{array}$ & $\begin{array}{c}\text { Lower } \\
\text { bound }\end{array}$ & $\begin{array}{c}\text { Upper } \\
\text { bound }\end{array}$ \\
\hline TAX & 59 & 0.805 & 1.13 & 0.44 & 2.87 \\
\hline ANT & 57 & 0.449 & 1.45 & 0.55 & 3.80 \\
\hline 5-FU/ & 22 & 0.805 & 1.13 & 0.44 & 2.87 \\
MTX & & & & & \\
\hline PBA & 20 & 0.222 & 1.89 & 0.68 & 5.28 \\
\hline CLA & 59 & 0.194 & 1.94 & 0.71 & 5.31 \\
\hline mAb & 35 & $0.043^{*}$ & 2.33 & 0.95 & 5.73 \\
\hline Other & 43 & 0.448 & 0.71 & 0.30 & 1.71 \\
CTx & & & & & \\
\hline HTx & 63 & 0.154 & 2.20 & 0.73 & 6.59
\end{tabular}

*Significant.

$\mathrm{TAX}=$ taxanes; ANT = anthracyclines; 5-FU = fluorouracil; MTX = methotrexate; PBA = platinum-based agents; CLA = DNA cross-link agents; $\mathrm{mAb}=$ monoclonal antibodies; $\mathrm{CTx}=$ chemotherapy; HTx = hormone therapy. late follow-up. For all other categories, we find radioembolization to be associated with severe toxicity in only $2 \%-10 \%$ of BRCLM patients. Our data indicate that this toxicity can be further reduced by administering radioembolization in 2 separate sessions. The summed incidence rate for SAEs was lower by about $15 \%$ in the group with SL radioembolization. Most importantly, there was no case of REILD in the SL group. Prior therapy with monoclonal antibodies emerges as an independent risk factor for high-grade toxicity in our cohort. In particular, prior administration of bevacizumab or trastuzumab significantly increased the risk for SAEs by 2.3-fold. Both of these agents are known to effectively suppress angiogenesis (19). Because antiangiogenic treatment is associated with increased risk for stasis or retrograde flow, we suppose that these phenomena might cause tissue damage due to inadvertently excessive microsphere loading in healthy liver or even adjacent organs $(20,21)$. Our data suggest that radioembolization should, whenever possible, be performed before administration of angiosuppressive therapy, so as to decrease the risk for SAEs.

We investigated clinical, radiologic, and laboratory findings regarding their prognostic value for OS. Of the included parameters, tumor burden of the liver and baseline transaminase toxicity each turned out to be independent predictors of patient survival. Disease burden of the liver has been previously described to be predictive for REILD or short survival after radioembolization in patients with hepatic and nonhepatic malignancies including breast cancer $(7,8,10,18)$. In our study, median survival was 41 wk (IQR, 35 wk) in patients with less than $25 \%$ replacement of liver parenchyma by tumor. Conversely, median survival was only $30 \mathrm{wk}$ (IQR, $36 \mathrm{wk}$ ) in patients with $25 \%-50 \%$ and $17 \mathrm{wk}(\mathrm{IQR}, 14 \mathrm{wk})$ in patients with $50 \%$ or more tumor burden. This difference underlines the need to carefully evaluate the suitability of patients with a high degree of liver involvement for radioembolization. The degree of tumor involvement is associated

TABLE 4

OS, TTP liver, and Response by ${ }^{18} \mathrm{~F}-\mathrm{FDG}$ PET or CA15-3

\begin{tabular}{lc}
\hline \multicolumn{1}{c}{ Response category } & Total or median \\
\hline OS & 81 \\
$n n$ & $35(41)$ \\
\hline After radioembolization (wk) & $7.2(7.3)$ \\
\hline After initial diagnosis $(\mathrm{y})$ & \\
\hline TP $_{\text {liver }}$ & 30 \\
\hline$n$ & $26(18)$ \\
\hline After radioembolization (wk) & 56 \\
\hline PET & $29(52 \%)$ \\
\hline$n$ & $27(48 \%)$ \\
\hline Responder & \\
\hline Nonresponder & 46 \\
CA15-3 & $28(61 \%)$ \\
\hline$n$ & $18(39 \%)$ \\
\hline Responder &
\end{tabular}

Given are total numbers (with percentages in parentheses) or median (with IQRs in parentheses). 
TABLE 5

Univariate Cox Regression Analysis for OS After Radioembolization

\begin{tabular}{|c|c|c|c|c|c|c|c|}
\hline \multirow[b]{2}{*}{ Variable } & \multirow[b]{2}{*}{ Median } & \multirow[b]{2}{*}{$\begin{array}{l}\text { Subcategories based } \\
\text { on rounded median }\end{array}$} & \multirow[b]{2}{*}{$n$} & \multirow[b]{2}{*}{$P$} & \multirow[b]{2}{*}{$\begin{array}{l}\text { Hazard } \\
\text { ratio }\end{array}$} & \multicolumn{2}{|c|}{$\begin{array}{l}95 \% \text { confidence } \\
\text { interval }\end{array}$} \\
\hline & & & & & & $\begin{array}{l}\text { Lower } \\
\text { bound }\end{array}$ & $\begin{array}{l}\text { Upper } \\
\text { bound }\end{array}$ \\
\hline \multirow[t]{2}{*}{ Prior local treatment } & & No & 61 & & 1.0 & & \\
\hline & & Yes & 20 & $0.152^{\star}$ & 0.66 & 0.38 & 1.16 \\
\hline \multirow[t]{2}{*}{ Treatment group } & & SL & 23 & & 1.0 & & \\
\hline & & WL & 58 & 0.938 & 1.02 & 0.60 & 1.75 \\
\hline \multirow[t]{3}{*}{ Tumor-to-liver ratio } & & $<25 \%$ & 49 & & 1.0 & & \\
\hline & & $25 \%-50 \%$ & 24 & $0.048^{\star}$ & 1.64 & 0.99 & 2.72 \\
\hline & & $\geq 50 \%$ & 8 & $<0.001^{*}$ & 6.36 & 2.74 & 14.79 \\
\hline \multirow{2}{*}{$\begin{array}{l}\text { Chemo }<3 \text { mo before } \\
\text { radioembolization }\end{array}$} & & No & 53 & & 1.0 & & \\
\hline & & Yes & 28 & $0.186^{\star}$ & 1.39 & 0.85 & 2.25 \\
\hline \multirow[t]{2}{*}{ Dose (GBq) } & 1.6 & $<1.6$ & 40 & 0.352 & 1.25 & 0.78 & 1.98 \\
\hline & & $\geq 1.6$ & 41 & & 1.0 & & \\
\hline \multirow[t]{2}{*}{ Baseline CA15-3 } & 137 & $<137$ & 40 & & 1.0 & & \\
\hline & & $\geq 137$ & 41 & $0.065^{\star}$ & 1.55 & 0.97 & 2.48 \\
\hline \multirow[t]{2}{*}{ Baseline transaminase toxicity } & & WNL & 61 & & 1.0 & & \\
\hline & & Grade $\geq 2$ & 20 & $0.001^{*}$ & 2.48 & 1.44 & 4.26 \\
\hline \multirow[t]{2}{*}{ Baseline GGT toxicity } & & WNL & 36 & & 1.0 & & \\
\hline & & Grade $\geq 2$ & 45 & $0.008^{*}$ & 1.89 & 1.18 & 3.03 \\
\hline \multirow[t]{2}{*}{ Baseline bilirubin toxicity } & & WNL & 73 & & 1.0 & & \\
\hline & & Any grade & 8 & 0.279 & 1.51 & 1.72 & 3.16 \\
\hline \multirow[t]{2}{*}{ Extrahepatic metastasis } & & No & 27 & & 1.0 & & \\
\hline & & Yes & 54 & 0.299 & 1.30 & 0.79 & 2.12 \\
\hline
\end{tabular}

${ }^{\star} P<0.20$, variables were included into multivariate analysis.

$\mathrm{WNL}=$ within normal limits.

with severity of liver damage after radioembolization as reported by Sangro et al. (8). Therefore, blood tests, including transaminase and bilirubin serum levels, are routinely obtained to estimate baseline liver function. We analyzed the prognostic values of baseline levels of AST, ALT, GGT, and bilirubin, and found a CTCAE toxicity grade 2 and greater for ALT or AST to be an independent predictor of short survival after radioembolization. Both extensive tumor involvement of the liver and high-grade transaminase toxicity indicate reduced liver capacity.
Radioembolization should thus be avoided in patients presenting with both risk factors.

The retrospective design of this study may have resulted in false low-toxicity findings arising from underreporting. However, the incidence of adverse events was similar to that reported in previous trials, especially for events with severity of grade 3 or higher. Our patients received no further liver-directed therapy until routine follow-up at 3 mo after radioembolization. However, we did not analyze the effect of subsequent treatments. Cancer-directed

TABLE 6

Multivariate Cox Regression Analysis of Selected Variables

\begin{tabular}{|c|c|c|c|c|}
\hline \multirow[b]{2}{*}{ Variable } & \multirow[b]{2}{*}{$n$} & \multicolumn{3}{|c|}{ Multivariate Cox regression } \\
\hline & & Hazard ratio & 95\% confidence interval & $P$ \\
\hline Total & 81 & & & \\
\hline Tumor-to-liver ratio $\geq 50 \%$ & 8 & 5.67 & $2.41-13.34$ & $<0.001^{*}$ \\
\hline Baseline transaminase toxicity grade $\geq 2$ & 20 & 2.15 & $1.21-3.80$ & $0.009^{*}$ \\
\hline
\end{tabular}




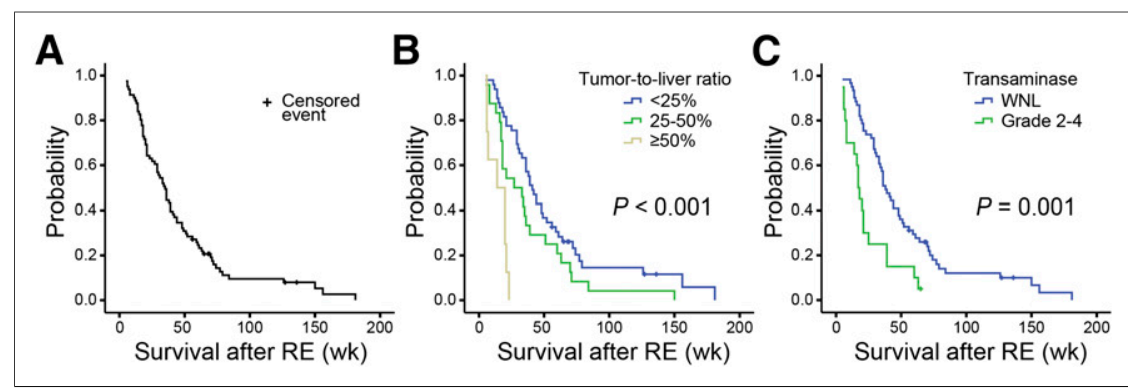

FIGURE 3. OS after radioembolization (RE) for entire study cohort (A), separated by tumor-toliver ratio $(B)$ and transaminase toxicity $(C)$. Log-rank $P$ is given. $W N L=$ within normal limits.
3. Chua TC, Saxena A, Liauw W, Chu F, Morris DL. Hepatic resection for metastatic breast cancer: a systematic review. Eur J Cancer. 2011;47:22822290.

4. Bangash AK, Atassi B, Kaklamani V, et al. ${ }^{90} \mathrm{Y}$ radioembolization of metastatic breast cancer to the liver: toxicity, imaging response, survival. $J$ Vasc Interv Radiol. 2007;18:621-628.

5. Coldwell DM, Kennedy AS, Nutting CW. Use of yttrium-90 microspheres in the treatment of unresectable hepatic metastases from breast cancer. Int J Radiat Oncol Biol Phys. 2007;69:800-804.

6. Haug AR, Tiega Donfack BP, Trumm C, et al. ${ }^{18} \mathrm{~F}-$ FDG PET/CT predicts survival after radioembolization of hepatic metastases from breast cancer. $J$ Nucl Med. 2012;53:371-377.

7. Sato KT, Lewandowski RJ, Mulcahy MF, et al. therapy after the 3 mo follow-up might have influenced survival in the late-line setting.

\section{CONCLUSION}

Radioembolization for BRCLM is associated with good treatment response and a low incidence of severe adverse events. The most favorable safety was encountered in patients with sequential lobar radioembolization and in patients without prior angiosuppressive therapy. A hepatic tumor burden of $50 \%$ or more and transaminase toxicity grade of 2 or greater at baseline independently indicate short survival.

\section{DISCLOSURE}

The costs of publication of this article were defrayed in part by the payment of page charges. Therefore, and solely to indicate this fact, this article is hereby marked "advertisement" in accordance with 18 USC section 1734. Alexander Haug, Marlies Michl, Philipp Paprottka, Sebastian Lehner, and Tobias Jakobs have received fees or travel-related expenses for events organized by SIRTEX Medical (Sydney, Australia) outside the submitted work. Philipp Paprottka and Tobias Jakobs have an advisory relationship with SIRTEX Medical outside the submitted work. This work is part of the doctoral thesis of Hanna Lechner. No other potential conflict of interest relevant to this article was reported.

\section{ACKNOWLEDGMENTS}

We acknowledge professional manuscript revision by Dr. Paul Cumming.

\section{REFERENCES}

1. Brewster AM, Hortobagyi GN, Broglio KR, et al. Residual risk of breast cancer recurrence 5 years after adjuvant therapy. J Natl Cancer Inst. 2008;100: 1179-1183.

2. Eichbaum MH, Kaltwasser M, Bruckner T, de Rossi TM, Schneeweiss A, Sohn C. Prognostic factors for patients with liver metastases from breast cancer. Breast Cancer Res Treat. 2006;96:53-62.
Unresectable chemorefractory liver metastases: radioembolization with ${ }^{90} \mathrm{Y}$ microspheres-safety, efficacy, and survival. Radiology. 2008;247:507-515.

8. Sangro B, Gil-Alzugaray B, Rodriguez J, et al. Liver disease induced by radioembolization of liver tumors: description and possible risk factors. Cancer. 2008;112:1538-1546.

9. Jakobs TF, Hoffmann RT, Fischer T, et al. Radioembolization in patients with hepatic metastases from breast cancer. J Vasc Interv Radiol. 2008;19:683690.

10. Gordon AC, Gradishar WJ, Kaklamani VG, et al. Yttrium-90 radioembolization stops progression of targeted breast cancer liver metastases after failed chemotherapy. J Vasc Interv Radiol. 2014;25:1523-1532.

11. Fendler WP, Ilhan H, Paprottka PM, et al. Nomogram including pretherapeutic parameters for prediction of survival after SIRT of hepatic metastases from colorectal cancer. Eur Radiol. 2015;25:2693-2700.

12. Fendler WP, Philippe Tiega DB, Ilhan H, et al. Validation of several SUV-based parameters derived from ${ }^{18} \mathrm{~F}$-FDG PET for prediction of survival after SIRT of hepatic metastases from colorectal cancer. J Nucl Med. 2013;54:1202-1208.

13. Karnofsky DA, Burchenal JH. The clinical evaluation of chemotherapeutic agents in cancer. In: MacLeod CM, ed. Evaluation of Chemotherapeutic Agents. New York, NY: Columbia University Press; 1949:196.

14. Kennedy A, Nag S, Salem R, et al. Recommendations for radioembolization of hepatic malignancies using yttrium-90 microsphere brachytherapy: a consensus panel report from the radioembolization brachytherapy oncology consortium. Int J Radiat Oncol Biol Phys. 2007;68:13-23.

15. Common terminology criteria for adverse events, version 4.0. EORTC website. http://www.eortc.be/services/doc/ctc/CTCAE_4.03_2010-06-14_QuickReference_5x7. pdf. Updated June 14, 2010. Accessed January 20, 2016.

16. Guadagni F, Ferroni P, Carlini S, et al. A re-evaluation of carcinoembryonic antigen (CEA) as a serum marker for breast cancer: a prospective longitudinal study. Clin Cancer Res. 2001;7:2357-2362.

17. Lee YT. Breast carcinoma: pattern of metastasis at autopsy. J Surg Oncol. 1983;23: 175-180.

18. Saxena A, Kapoor J, Meteling B, Morris DL, Bester L. Yttrium-90 radioembolization for unresectable, chemoresistant breast cancer liver metastases: a large single-center experience of 40 patients. Ann Surg Oncol. 2014;21: 1296-1303.

19. Izumi Y, Xu L, di Tomaso E, Fukumura D, Jain RK. Tumour biology: herceptin acts as an anti-angiogenic cocktail. Nature. 2002;416:279-280.

20. Lam MG, Banerjee S, Louie JD, et al. Root cause analysis of gastroduodenal ulceration after yttrium-90 radioembolization. Cardiovasc Intervent Radiol. 2013;36: 1536-1547.

21. Ahmadzadehfar H, Sabet A, Meyer C, Habibi E, Biersack HJ, Ezziddin S. The importance of Tc-MAA SPECT/CT for therapy planning of radioembolization in a patient treated with bevacizumab. Clin Nucl Med. 2012;37: 1129-1130. 